\title{
Analysis of salivary phenotypes of generalized aggressive and chronic periodontitis through nuclear magnetic resonance-based metabolomics
}

Federica Romano*, Gaia Meoni ${ }^{\dagger \dagger}$, Valeria Manavella ${ }^{*}$, Giacomo Baima*, Leonardo Tenori ${ }^{\dagger \dagger}$, Stefano Cacciatore $^{\S}$, Mario Aimetti*

"Department of Surgical Sciences, C.I.R. Dental School, University of Turin, Turin, Italy.

${ }^{\dagger}$ Magnetic Resonance Center (CERM), University of Florence, Sesto Fiorentino, Italy.

${ }^{*}$ Department of Experimental and Clinical Medicine, University of Florence, Sesto Fiorentino, Italy.

${ }^{\S}$ Department of Surgery \& Cancer, Imperial College, London, UK and International Centre for Genetic Engineering and Biotechnology, Cancer Genomics Group, Cape Town, South Africa.

Federica Romano and Gaia Meoni contributed equally to this article.

\section{Corresponding author:}

Prof. Mario Aimetti, Section of Periodontology, C.I.R. Dental School, Via Nizza 23010126 Turin (Italy). Phone: +390116331543 email: mario.aimetti@unito.it Fax: +390116331506 (Fax number and e-mail can be published).

\section{Word count: 3460}

This is the author manuscript accepted for publication and has undergone full peer review but has not been through the copyediting, typesetting, pagination and proofreading process, which may lead to differences between this version and the Version of Record. Please cite this article as doi: 10.1002/JPER.18-0097.

This article is protected by copyright. All rights reserved. 


\title{
Number of Tables: 2
}

\section{Number of Figures: 4}

\section{Number of references: 46}

Running title: Salivary metabolomics in periodontitis

One sentence summary: Metabolomic analysis of saliva discriminates healthy individuals from periodontitis patients, irrespectively of the aggressive or chronic periodontitis profile.

\begin{abstract}
Background: Recent findings about the differential gene expression signature of periodontal lesions have raised the hypothesis of distinctive biological phenotypes expressed by generalized chronic periodontitis (GCP) and generalized aggressive periodontitis (GAgP) patients. Therefore, this crosssectional investigation was planned, primarily, to determine the ability of nuclear magnetic resonance (NMR) spectroscopic analysis of unstimulated whole saliva to discriminate GCP and GAgP diseasespecific metabolomic fingerprint and, secondarily, to assess potential metabolites discriminating periodontitis patients from periodontally healthy individuals (HI).
\end{abstract}

Methods: NMR-metabolomics spectra were acquired from salivary samples of patients with a clinical diagnosis of GCP $(n=33)$ or GAgP $(n=28)$ and from HI $(n=39)$. The clustering of HI, GCP and GAgP patients was achieved by using a combination of the Principal Component Analysis and Canonical Correlation Analysis on the NMR profiles. Results: These analyses revealed a significant predictive accuracy discriminating $\mathrm{HI}$ from GCP, and discriminating $\mathrm{HI}$ from GAgP patients (both 81\%). In contrast, the GAgP and GCP saliva samples seem to belong to the same metabolic space (60\% predictive accuracy). Significantly lower levels $(P<0.05)$ of pyruvate, $N$-acetyl groups and 
lactate and higher levels $(P<0.05)$ of proline, phenylalanine, and tyrosine were found in GCP and GAgP patients compared with HI.

Conclusions: Within the limitations of this study, CGP and GAgP metabolomic profiles were not unequivocally discriminated through a NMR-based spectroscopic analysis of saliva.

Keywords: biomarkers, metabolomics, magnetic resonance spectroscopy, periodontal diseases, saliva.

\section{Introduction}

Periodontitis is a multifactorial, chronic, inflammatory disease that leads to loss of periodontal attachment to the root surface and alveolar bone resorption and, if untreated, ultimately results in tooth exfoliation. ${ }^{1}$ It is widely accepted that dysbiosis within the human dental plaque biofilm is the primary initiator of periodontitis ${ }^{2}$, even though the extent and severity of tissue destruction appear to be host-mediated. ${ }^{3,4}$

Periodontitis can have heterogeneous clinical presentations. The traditional classification recognizes two major forms of periodontitis, chronic periodontitis (CP) and aggressive periodontitis ( $\mathrm{AgP})$, differing in rate of progression, prognosis and need for specific treatment approaches. ${ }^{5}$ At the present, the diagnosis $\mathrm{AgP}$ and $\mathrm{CP}$ is primarily based on clinical examination and radiographic parameters. ${ }^{5}$ No clinical, histopathological or microbiological assessment provides an unequivocal discrimination between the two conditions. ${ }^{6,7}$ For this reason, there is a strong effort to discover specific molecular arrays as a diagnostic tool to differentiate $\mathrm{CP}$ and $\mathrm{AgP}$ by oral-health professionals. ${ }^{8}$

Several molecules in the oral fluids, namely gingival crevicular fluid (GCF) and saliva, have been investigated so far in the attempt to provide detailed understanding of the biochemical network of periodontal tissue destruction. ${ }^{9}$ Saliva is particularly promising as it contains locally produced 
proteins, as well as other molecules from the systemic circulation. ${ }^{8}$ Furthermore, its collection is noninvasive, rapid and inexpensive. ${ }^{10}$ Salivary diagnostics has already proved efficient in identifying alterations in oral and systemic health status. ${ }^{11,12}$ Even so, various challenges persist regarding the use of saliva as a medium for an accurate and cost-effective detection of periodontitis, mainly due to the lack of specific markers of disease. ${ }^{13}$

Metabolomics is a newly emerging field of research dealing with the high-throughput identification and quantification of the whole ensemble of metabolites (small molecules; $<1500 \mathrm{Da}$ ) in a cell, tissue, body fluids or ecological systems. ${ }^{14}$ The metabolomics profiling reflects the dynamic response of a living system to genetic modification and physiological, pathological, and developmental stimuli. ${ }^{15}$ Thus, metabolomics offers the potential for a holistic approach to an individualized, patient-centered medicine.

Compared with other high throughput approaches, the main benefit of metabolomics analysis resides in its ability to take a snap at the very end-point of all the complex causal pathways driving periodontal pathogenesis. Small molecules derived from the dysbiotic community and host tissue breakdown, targeted by metabolomics, are potentially able to reflect the real-time molecular phenotype of the disease. ${ }^{9}$ At the same time, it has been proven that saliva is a stable biofluid and that a clear individual metabolic phenotype can be revealed using saliva samples. ${ }^{16}$

Untargeted metabolomics by nuclear magnetic resonance (NMR) spectroscopy or mass spectrometry (MS) has been previously employed to differentiate healthy and periodontally diseased individuals through the pattern recognition analysis of saliva and GCF, since this approach has the advantage to maximize the number of metabolites detected, including chemical unknowns. ${ }^{17-19}$ Although some studies provided promising preliminary outcomes regarding the detection of some panels of discriminant metabolites, further trials with larger sample sizes are needed in order to add consistency and external validity to these results. ${ }^{17-20}$ 
To the best of our knowledge, the possibility to employ the NMR-based metabolomics analysis to discriminate $\mathrm{CP}$ and $\mathrm{AgP}$ remains to explore. This pilot study was designed to test the hypothesis that untargeted metabolomic analysis of saliva could differentiate the biochemical signatures of the generalized forms of chronic periodontitis (GCP) and aggressive periodontitis (GAgP). The secondary aim was related to the detection of a differentially expressed array of metabolites that could be further investigated as potential biomarkers for the development of a rapid diagnostic tool for periodontitis.

\section{Material and Methods}

The protocol of this cross-sectional study was approved by the Institutional Ethical Review Board (protocol number 1503/2016) and the study was conducted in accordance with the Helsinki Declaration. Written informed consent was obtained from all participants. The study was reported according to the STROBE guidelines.21.

\section{Study population}

The sample size was set at 100 individuals based on the results of previous studies ${ }^{19,22}$ and the pilot nature of this study. A total of 33 patients with GCP (mean age: $50.5 \pm 8.9$ years, $63.6 \%$ males and 15.2\% smokers), 28 patients with GAgP (mean age: $31.1 \pm 4.6$ years, $64.3 \%$ males and $14.3 \%$ smokers) and 39 periodontally healthy individuals (mean age: $46.6 \pm 8.2$ years, $64.1 \%$ males and $15.4 \%$ smokers) were consecutively recruited from among individual seeking oral health consultation at the C.I.R. Dental School, University of Turin (Italy) from January to September 2017. After being screened, participants were balanced with respect to gender, and smoking habits.

Exclusion criteria included less than 20 teeth; antibiotic intake within the previous 3 months, periodontal treatment during the past 6 months, abnormal salivary function, diagnosis of any disease in oral and hard tissues and other systemic conditions that could influence periodontal status and 
metabolomic profile (e.g. diabetes mellitus and metabolic syndromes), regular alcohol consumption, pregnancy and lactation. ${ }^{23}$

\section{Determination of periodontal status and saliva collection}

All participants underwent a periodontal examination by two experienced clinicians (V.M, F.R) who were previously trained and calibrated for the periodontal examination and saliva sampling. A set of full-mouth periapical radiographs was taken for each patient. Presence/absence of plaque (PI), presence/absence of bleeding on probing (BoP), probing depth (PD), and clinical attachment level (CAL) were measured at six sites around each tooth by manual probing ${ }^{\prime \mid}$. Inter-examiner reliability was determined by having each examiner made dual measurements along with those of the Project Director (M.A) on 15 non-study patients, and intra-examiner reproducibility was assessed by taking replicate measurements on the same patients with an interval of 24 hours between the first and the second recording. The percentage of agreement within $1 \mathrm{~mm}$ of PD and CAL ranged between $94 \%$ and $97 \%$.

Patients with GCP and GAgP and healthy controls were diagnosed based on the current classification of the 1999 International Workshop for the Classification of Periodontal Diseases and Conditions ${ }^{5}$ and met the following criteria. GCP patients had $\geq 30 \%$ of sites with PD and CAL $>5 \mathrm{~mm}$, and presence of BoP. ${ }^{5}$ Patients in the GAgP group were $<35$ years of age, and had at least six permanent first molars and/or incisors with at least one site with $\mathrm{PD}$ and CAL $>5 \mathrm{~mm}$ as well as a minimum of six teeth other than first molars and incisors also presenting at least one site each with PD and CAL $>5$ $\mathrm{mm} .{ }^{24}$ Other factors such as family aggregation, rapid progression and the relationship between local factors and periodontal destruction were also considered. ${ }^{5}$ The control group comprised healthy

"

This article is protected by copyright. All rights reserved. 
individuals (HI) with PD and $\mathrm{CAL} \leq 3 \mathrm{~mm}$ at all sites on all teeth, no radiographic evidence of alveolar bone loss, and $<15 \%$ of sites presenting $\mathrm{BoP}^{24}$

At least 24 hours after periodontal measurements to avoid blood contamination, unstimulated whole saliva was obtained by all study subjects between 8:00 and 10:00 am using standard techniques as described by Silwood et al. ${ }^{25}$. Briefly, all subjects were advised to refrain from using mouthwash and brushing their teeth at least $1 \mathrm{~h}$ before sample collection. Each subject was instructed not to force salivation, to allow saliva to be collected in the mouth, and let the saliva drain into a sterile graduated tube for $10 \mathrm{~min}$. About $1 \mathrm{ml}$ of saliva was collected from every patient and immediately frozen.

\section{NMR sample preparation}

Frozen saliva samples were thawed at room temperature and were centrifuged $(5000 \times g$ for a period of $30 \mathrm{~min}$ at $\left.4{ }^{\circ} \mathrm{C}\right)$ to remove debris. A total of $300 \mu \mathrm{l}$ of sodium phosphate buffer $\left(70 \mathrm{mM} \mathrm{Na}_{2} \mathrm{HPO}_{4}\right.$; $20 \%(\mathrm{v} / \mathrm{v}){ }^{2} \mathrm{H}_{2} \mathrm{O} ; 6.15 \mathrm{mM} \mathrm{NaN}_{3} ; 6.64 \mathrm{mM}$ sodium trimethylsilyl [2,2,3,3- $\left.{ }^{2} \mathrm{H}_{4}\right]$ propionate (TMSP); $\mathrm{pH}$ 7.4) was immediately added to $300 \mu \mathrm{l}$ of each sample, and the mixture was homogenized by vortexing for $30 \mathrm{~s} . \mathrm{NaN}_{3}$ was added as a preservative to ensure that metabolites were not generated or consumed due to bacteria present in the saliva during the time of preparation of the samples or of the acquisition of NMR spectra. A total of $450 \mu \mathrm{l}$ of this mixture was transferred into a $4.25 \mathrm{~mm}$ NMR tubeף for analysis.

\section{NMR spectral acquisition}

NMR spectra for all samples were acquired using a spectrometer ${ }^{\#}$ operating at $600.13 \mathrm{MHz}$ proton Larmor frequency equipped with a $5 \mathrm{~mm} \mathrm{CPTCI}{ }^{1} \mathrm{H}-{ }^{13} \mathrm{C}-{ }^{31} \mathrm{P}$ and ${ }^{2} \mathrm{H}$-decoupling cryoprobe including a $\mathrm{z}$ axis gradient coil, an automatic tuning-matching (ATM) and an automatic sample changer. A BTO

\footnotetext{
" Bruker BioSpin srl, Milan, Italy.

\# Bruker BioSpin srl, Milan, Italy.
}

This article is protected by copyright. All rights reserved. 
2000 thermocouple served for temperature stabilization at the level of approximately $0.1 \mathrm{~K}$ at the sample. Before measurement, samples were kept for at least 3 min inside the NMR probehead, for temperature equilibration $(300 \mathrm{~K})$. For each saliva sample, a ${ }^{1} \mathrm{H}-\mathrm{NMR}$ spectrum was acquired using the pulse sequence NOESY-presat with 64 free induction decay (FID) collected into 65536 data points over a spectral width of $12019 \mathrm{~Hz}$, relaxation delay (RD) of $4 \mathrm{~s}$ and mixing time of $0.1 \mathrm{~s}$.

\section{Spectral processing and analysis}

Free induction decays were multiplied by an exponential function equivalent to a $1.0 \mathrm{~Hz}$ linebroadening factor before applying Fourier transform. Transformed spectra were automatically corrected for phase and baseline distortions and calibrated using a RMN processing software ${ }^{* *}$. Spectra were aligned by calibrating the TMSP peak at $0.00 \mathrm{ppm}$. Each $1 \mathrm{D}$ spectrum in the spectral ranges $0.2-4.3$ and $6.6-10.0 \mathrm{ppm}$ was segmented into $0.02 \mathrm{ppm}$ chemical shift bins, and the corresponding spectral areas were integrated using a specific software program $\uparrow \uparrow$. The binning procedure is a mean to reduce the number of total variables, to compensate for subtle signal shifts, and filter noise in the spectra, making the analysis more robust and reproducible. ${ }^{26,27}$ The total spectral area was calculated on the bins and total area normalization was carried out on the data prior to pattern recognition.

\section{Statistical analysis}

All data analyses were performed blindly using R statistical package. Significance difference among the clinical groups was calculated using analysis of variance for clinical data and post hoc significance of differences between pairs of comparisons was determined using Fisher least significant difference procedure.

\footnotetext{
${ }^{* *}$ Tospin version 2.1, Bruker BioSpin srl, Milan, Italy.

† Amix software, version 3.8.4, Bruker BioSpin, Milan, Italy.
}

This article is protected by copyright. All rights reserved. 
Multivariate statistical analysis tools were applied to study the metabolomics profiles of GCP, GAgP and HI groups. ${ }^{28}$ The supervised statistical procedure applied for data reduction and classification was a combination of Principal Component Analysis (PCA) and canonical correlation analysis (CA) on the PCA scores. K-nearest neighbors $(\mathrm{kNN})$ learning method $(\mathrm{k}=5)$ applied on the CA scores was used to predict test samples. The global accuracy for classification was assessed by means of a Monte Carlo cross-validation scheme. Twenty-two metabolites, corresponding to well defined and resolved peaks in the spectra, were assigned. Signal identification was performed using a library of NMR spectra of pure organic compounds, public databases (e.g. HMBD) storing reference. The relative concentrations of the various metabolites in the different spectra were calculated by spectral fitting and integration of the signal area using in-house scripts ${ }^{\$ 2} .{ }^{29}$ The Wilcoxon test was used for the determination of the statistically relevant metabolites. False discovery rate correction (FDR) was applied using the Benjamini and Hochberg method ${ }^{30}$ : an adjusted $P$-value $\leq 0.05$ was considered statistically significant. The changes in metabolites levels between periodontitis and healthy controls spectra were calculated as the $\log _{2}$ fold change $(\mathrm{FC})$ ratio of the normalized median intensities of the corresponding signals in the spectra of the two groups. A statistical software program was used for pathway analysis ${ }^{\S \S} .31$

\section{Results}

\section{Characteristics of the study population}

Demographic and clinical characteristics of the 100 participants according to the periodontal diagnosis are shown in supplementary Table 1 in the online Journal of Periodontology. The mean age in the GAgP group was significantly less than the other two groups, whereas ages were similar for the

\footnotetext{
${ }^{\ddagger \ddagger}$ Matlab and Statistics Toolbox, Mathworks Inc., Natick, Massachusetts, USA

$\S \S$ MetaboAnalyst version 3.0, www.metaboanalyst.ca
}

This article is protected by copyright. All rights reserved. 
GCP and HI groups $(P>0.05)$. Smokers in all the three clinical groups smoked less than 10 cigarettes a day (range 5 to 8 ).

As expected, the mean Full-Mouth Plaque Scores (FMPS), Full-Mouth Bleeding Scores (FMBS), PD, and CAL values were statistically significantly higher in patients with GAgP and GCP compared with the HI; all reached $P<0.001$. When GCP and GAgP groups were compared, the only statistically significant difference found was in FMPS $(P<0.001)$.

\section{Metabolomic profiling of saliva}

The clustering of HI, GCP and GAgP patients was achieved by using PCA/CA on the ${ }^{1} \mathrm{H}-\mathrm{NMR}$ profiles of saliva samples (Fig. 1). These analyses revealed $81 \%$ predictive accuracy discriminating HI from GCP, and 81\% discriminating HI from GAgP affected patients (Fig. 2A,B). Permutation test (number of permutations $=1000)$ results showed statistically significant classification accuracy $(P<$ 0.001). The statistical model applied proved to be effective to discriminate HI from GCP and GAgP patients, while the same statistical approach was not effective to discriminate GCP from the GAgP counterpart (60\% predictive accuracy). Indeed, GAgP and GCP saliva samples seem belonging to the same metabolic space (Fig. 3). The predictive accuracy of these models did not change when smokers were excluded from the analysis ( $81 \% \mathrm{HI}$ vs. GCP, $81 \% \mathrm{HI}$ vs. GAgP, $60 \% \mathrm{GAgP}$ vs. GCP).

\section{Metabolites contributing to periodontal disease}

The discrimination obtained between saliva samples of GCP and GAgP patients compared with HI, also demonstrated the existence of an altered metabolism in patients affected from periodontitis. Assigned signals in NMR spectra were integrated to obtain the concentration of metabolites in arbitrary units. By comparing the spectra of the saliva samples of periodontitis patients with $\mathrm{HI}$, it results that GCP patients are characterized by lower levels $(P<0.05)$ of pyruvate, $N$-acetyl groups and lactate, and higher levels $(P<0.05)$ of proline, phenylalanine, isoleucine, valine and tyrosine, as summarized in Fig. 4A and Table 1. Compared with HI, GAgP

This article is protected by copyright. All rights reserved. 
patients are characterized by lower levels $(P<0.05)$ of pyruvate, $N$-acetyl groups and lactate and sarcosine, and higher levels $(P<0.05)$ of formate, phenylalanine and tyrosine (Fig. 4B). A simplified list of the most contributing metabolic pathways is reported in Table 2. The analysis showed alteration in biochemical pathways like phenylalanine metabolism (phenylalanine, pyruvic acid, tyrosine, lactic acid) and pyruvate metabolism (pyruvic acid and lactic acid). The analysis was calculated based on adjusted $P$-value $(P<0.05)$ of the pathway enrichment analysis and an "Impact" (calculated from pathway topology analysis) equal to or greater than 0.1 was considered significant.

\section{Discussion}

The present study was designed to test the ability of NMR-based metabolomics to differentiate the biochemical signatures of GCP and GAgP in human saliva. To this purpose, gingivitis and localized manifestations of periodontitis were pointedly excluded because of the risk of flawing the results, and only periodontally $\mathrm{HI}$ were selected as controls. However, while corroborating substantial differences between pathological and healthy periodontal conditions, the multivariate analysis of NMR spectra did not provide a significant discrimination between the GCP and GAgP metabolomics profiles. The latter finding is in agreement with an increasing body of evidence and confirms that it is almost impossible to use the term $\mathrm{AgP}$ as long as there is no proper way to diagnose the disease. ${ }^{32}$ Indeed, the discrimination between $\mathrm{CP}$ and $\mathrm{AgP}$ is not supported by sufficiently distinct histological, microbiological, immunological or genetic foundations. ${ }^{33-37}$ Moreover, microbiome exhibits conserved metabolic and virulence gene expression profiles despite the inter-individual differences in the disease phenotype. ${ }^{38}$ This may suggest that what distinguishes AgP from $\mathrm{CP}$ are dissimilarities in the immune-inflammatory host response ${ }^{39}$ or, as advocated by Van der Velden ${ }^{32}$, a difference in the degree of bacterial invasiveness. It is unlikely that the sole analysis of the metabolites in oral fluids can detect any pathognomonic benchmarks. Presumably, as far as all new high-throughput technologies have proven the existence of several molecular signatures in distinct periodontal patients $^{35}$, the traditional binomial classification seems not to fit this emerging heterogeneity anymore.

This article is protected by copyright. All rights reserved. 
New models need to be hypothesized and tested, but due to the disease complexity a simultaneous multiomics approach should be elected.

The secondary goal was assessing the potential of oral fluid-based metabolomics to provide robust molecular biomarkers for periodontal diagnosis. Chronic periodontal infections activate the patient's host response to liberate a myriad of metabolic products at the interface between the tooth and the periodontal pocket. ${ }^{13}$ The discrimination between the salivary samples from periodontitis patients, irrespective of the type of disease, and HI strengthens the evidence of a metabolomics trace of periodontitis in human saliva. ${ }^{17}$ The Human Metabolome Database reports about 800 metabolites detected in saliva. However, according to similar studies, the NMR spectral profiles of this set of subjects are dominated by the signals of 20-30 molecules. ${ }^{16-18}$ The values of the relative concentrations of saliva metabolites were estimated through the integration of the signals in the NMR spectra and were found consistent with the results and the biological interpretation of a previous publication of this same group. ${ }^{18}$ The significantly reduced levels of lactate detected in the saliva of patients with GCP are partially explained by its conversion to acetate and propionate by some of the most prevalent periodontal bacterial species. ${ }^{40,41}$ This may reflect on the pyruvate concentrations as the result of the substrate depletion of the L-lactate dehydrogenase. The levels of proline, phenylalanine, isoleucine, valine and tyrosine were higher in the saliva samples of patients with GCP with respect to $\mathrm{C}$, as the amounts (not significant) of fatty acids, dipeptides and monosaccharide. This parallel up-regulation of the lipase, protease and glycosidase activities found in periodontitis is responsible for the overall tissue degradation and offers an ideal environment for bacterial proliferation and immune cells migration. ${ }^{42}$

The results of the present study should be interpreted with caution, as there are some limitations. One of the major problems with oral metabolites is that there is no way to determine their true origin. They could be essential constituent of the patient unique saliva, they could derive from the breakdown of the host tissues as from the bacterial communities, even from the supragingival plaque. Kuboniwa et 
al. ${ }^{22}$ performed supragingival scaling prior to sample collection and found that the discriminating ability of their model was significantly improved. By the way, in this study a scaling session was not performed; being able to find differences in a largely noisy environment could have more impact on the development of a rapid, noninvasive diagnostic tool.

Furthermore, the groups were matched for gender and smoking habits, yet not for age. This was not possible due to the difference in the age of onset of the two clinical forms of periodontitis, therefore GAgP patients were in average younger than GCP patients and the control group was comparable for age only with GCP individuals. Although there are no NMR-based metabolomics studies on the effect of aging on saliva, it is well-known that aging has a drastic effect on the serum metabolome. ${ }^{43,44}$ Concentrations of certain small molecule metabolites in saliva, including some hormones and many pharmaceuticals and drugs of abuse, are known to correlate quantifiably with concentrations in serum. Nonetheless, data from a companion study demonstrated that the salivary NMR fingerprint had low discrimination accuracy between young (14 - 40 years) and elderly ( $58-73$ years). ${ }^{18}$

Finally, due to the limited number of light smokers within this subset, the effect of smoking habits on metabolomics profiling was not specifically analyzed. However, when smokers were excluded from the analysis, the discrimination accuracy of the predicting models remained unchanged. It was thus plausible to exclude smoking as a confounding factor. This finding corroborates previous data demonstrating that light smoking had a negligible effect on the salivary profile. ${ }^{18}$

A major challenge in clinical periodontology is to find a reliable molecular marker of tissue destruction with high sensitivity, specificity and utility. At present, there is still a certain level of noise in metabolites fluctuation occurring in the periodontal microenvironment during the pathogenesis of periodontitis, that is not currently understood. ${ }^{45}$ The hurdle in identifying neat pathological phenotypes is due to the intrinsic heterogeneity of periodontal diseases and the inherent complexity underlying. ${ }^{35,46}$ Presumably, the main concern a clinician should have is not about discriminating 
between GCP and GAgP, but about the prompt detection of active or inactive phases of supporting tissue breakdown. Future endeavors of salivary biomarkers inquiries should be hence directed towards the real time assessment of disease activity and the molecular characterization of different phenotypes of severe periodontitis. Regarding this issue, metabolomics could be of most interest in future research directions.

\section{Conclusions}

This cross-sectional investigation provided the evidence that NMR-based metabolomics failed to detect an unequivocal biochemical signature discriminating GCP from GAgP. The absence of evidence is not automatically evidence of the absence, but this finding adds consistency to the quest to redefine the current classification of periodontitis. Conversely, the successful differentiation between healthy and diseased individuals corroborated the sensibility of metabolomics profiling as a source of potential panels of biomarkers for molecular diagnostics. Nonetheless, the complex multifactorial etiology of periodontitis will require large clinical trials bringing together a multiomics assessment of saliva to properly address this issue.

\section{Acknowledgments}

The authors received no financial support and declare no potential conflicts of interest with respect to the authorship and/or publication of this article.

This article is protected by copyright. All rights reserved. 


\section{REFERENCES}

1. Kornman KS. Mapping the pathogenesis of periodontitis: a new look. J Periodontol 2008;79(Suppl. 8):1560-1568.

2. Roberts FA, Darveau RP. Microbial protection and virulence in periodontal tissue as a function of polymicrobial communities: symbiosis and dysbiosis. Periodontol 2000 2015;69:18-27.

3. Bartold PM, Van Dyke TE. Periodontitis: a host-mediated disruption of microbial homeostasis. Unlearning learned concepts. Periodontol 2000 2013;62:203-217.

4. Papapanou PN. Commentary: advances in periodontal disease epidemiology: a retrospective commentary. J Periodontol 2014;85:877-879.

5. Armitage GC. Development of a classification system for periodontal diseases and conditions. Ann Periodontol 1999;4:1-6.

6. Armitage GC. Comparison of the microbiological features of chronic and aggressive periodontitis. Periodontol 2000 2010;53:70-88.

7. Ji S, Choi Y. Point-of-care diagnosis of periodontitis using saliva: technically feasible but still a challenge. Front Cell Infect Microbiol 2015;5:65.

8. Buduneli N, Kinane DF. Host-derived diagnostic markers related to soft tissue destruction and bone degradation in periodontitis. $J$ Clin Periodontol 2011;38(Suppl. 11):85-105.

9. Barros SP, Williams R, Offenbacher S, Morelli T. Gingival crevicular fluid as a source of biomarkers for periodontitis. Periodontol 2000 2016;70:53-64.

10. Kinney JS, Morelli T, Braun T, et al. Saliva/pathogen biomarker signatures and periodontal disease progression. $J$ Dent Res 2011;90:752-758.

11. Zhang Y, Sun J, Lin CC, Abemayor E, Wang MB, Wong DTW. The emerging landscape of salivary diagnostics. Periodontol 2000 2016;70:38-52.

This article is protected by copyright. All rights reserved. 
12. Rathnayake N, Gieselmann DR, Heikkinen AM, Tervahartiala T, Sorsa T. Salivary DiagnosticsPoint-of-Care diagnostics of MMP-8 in dentistry and medicine [published online ahead of print January 20, 2017]. Diagnostics (Basel); doi: 10.3390/diagnostics7010007.

13. Giannobile WV. Salivary diagnostics for periodontal diseases. J Am Dent Assoc 2012;143(Suppl. 10):6-11.

14. German JB, Hammock BD, Watkins SM. Metabolomics: building on a century of biochemistry to guide human health. Metabolomics 2005;1:3-9.

15. Clark J, Haselden JN. Metabolic profiling as a tool for understanding mechanisms of toxicity. Toxicol Pathol 2008;36:140-147.

16. Wallner-Liebmann S, Tenori L, Mazzoleni A, et al. Individual human metabolic phenotype analyzed by (1)H NMR of saliva samples. J Proteome Res 2016;15:1787-1793.

17. Barnes VM, Ciancio SG, Shibly O, et al. Metabolomics reveals elevated macromolecular degradation in periodontal disease. J Dent Res 2011;90:1293-1297.

18. Aimetti M, Cacciatore S, Graziano A, Tenori L. Metabonomic analysis of saliva reveals generalized chronic periodontitis signature. Metabolomics 2012;8:465-474.

19. Ozek NS, Zeller I, Renaul DE, et al. Differentitation of chronic and aggressive periodontitis by FTIR spectroscopy. $J$ Dent Res 2016;95:1472-1478.

20. Mikkonen JJW, Singh SP, Herrala M, Lappalainen R, Myllymaa S, Kullaa AM. Salivary metabolomics in the diagnosis of oral cancer and periodontal diseases. $J$ Periodontal Res 2016;51:431-437.

21. von Elm E, Altman DG, Egger M, Pocock SJ, Gotzsche PC, Vandenbroucke JP. The strengthening the reporting of observational studies in epidemiology (STROBE) statement: guidelines for reporting observational studies. Lancet 2010; 370:1453-1457.

22. Kuboniwa M, Sakanaka A, Hashino E, Bamba T, Fukusaki E, Amano A. Prediction of periodontal inflammation via metabolic profiling of saliva. J Dent Res 2016;95:1381-1386.

This article is protected by copyright. All rights reserved. 
23. Guasch-Ferré M, Hruby A, Toledo E, et al. Metabolomics in prediabetes and diabetes: a systematic review and meta-analysis. Diabetes Care 2016;39:833-846.

24. Ertugrul AS, Sahin H, Dikilitas A, Alpaslan N, Bozoglan A. Comparison of CCL28, interleukin-8, interleukin- $1 \mathrm{~b}$ and tumor necrosis factor-alpha in subjects with gingivitis, chronic periodontitis and generalized aggressive periodontitis. J Periodont Res 2013; 48:44-51.

25. Silwood CJL, Lynch EJ, Seddon S, Sheerin A, Claxson AWD, Grootveld MC. ${ }^{1}$ H-NMR analysis of microbial-derived organic acids in primary root carious lesions and saliva. NMR Biomed $1999 ; 12: 345-356$.

26. Holmes E, Foxall PJ, Nicholson JK, et al. Automatic data reduction and pattern recognition methods for analysis of $1 \mathrm{H}$ nuclear magnetic resonance spectra of human urine from normal and pathological states. Anal Biochem 1994;220:284-296.

27. Spraul M, Neidig P, Klauck U, et al. Automatic reduction of NMR spectroscopic data for statistical and pattern recognition classification of samples. J Pharm Biomed Anal 1994;12:12151225.

28. Madsen R, Lundstedt T, Trygg J. Chemometrics in metabolomics--a review in human disease diagnosis. Anal Chim Acta 2010;659:23-33.

29. Wishart DS. Quantitative metabolomics using NMR. TrAC Trends Anal Chem 2008;27:228-237.

30. Benjamini Y, Hochberg Y. On the adaptive control of the false discovery rate in multiple testing with independent statistics. $J$ Educ Behav Stat 2000;25:60-83.

31. Xia J, Sinelnikov IV, Han B, Wishart DS. MetaboAnalyst 3.0--making metabolomics more meaningful. Nucleic Acid Res 2015;43:W251-257.

32. Van der Velden U. What exactly distinguishes aggressive from chronic periodontitis: is it mainly a difference in the degree of bacterial invasiveness? Periodontol 2000 2017;75:24-44.

This article is protected by copyright. All rights reserved. 
33. Nibali L, D’Aiuto F, Ready D, Parkar M, Yahaya R, Donos N. No association between $A$. actinomycetemcomitans or $P$. gingivalis and chronic or aggressive periodontitis diagnosis. Quintessence Int 2012;43:247-254.

34. Kebschull M, Guarnieri P, Demmer RT, Boulesteix AL, Pavlidis P, Papapanou PN. Molecular differences between chronic and aggressive periodontitis. J Dent Res 2013; 92:1081-1088.

35. Loos BG, Papantonopoulos G. Molecular biotypes for periodontal diseases? J Dent Res 2013;92:1056-1057.

36. Könönen E, Müller HP. Microbiology of aggressive periodontitis. Periodontol 2000 2014;65:4678.

37. Ziukaite L, Slot DE, Loos BG, Coucke W, Van der Weijden GA. Family history of periodontal disease and prevalence of smoking status among adult periodontitis patients: a cross-sectional study. Int J Dent Hyg 2017;15:e28-e34.

38. Jorth P, Turner KH, Gumus P, Nizam N, Buduneli N, Whiteley M. Metatranscriptomics of the human oral microbiome during health and disease. mBio 2014;5:e01012-01014.

39. Champagne CME, Buchanan W, Reddy MS, Preisser JS, Beck JD, Offenbacher S. Potential for gingival crevice fluid measures as predictors of risk for periodontal diseases. Periodontol 2000 2003;31:167-180.

40. Distler W, Kroncke, A. Acid formation by mixed cultures of dental plaque bacteria Actinomyces and Veillonella. Arch Oral Biol 1981;26:123-126.

41. Traudt M, Kleinberg I. Stoichiometry of oxygen consumption and sugar, organic acid and amino acid utilization in salivary sediment and pure cultures of oral bacteria. Arch Oral Biol 1996;41:965978.

42. Barnes VM, Teles R, Trivedi HM, et al. Acceleration of purine degradation by periodontal diseases. $J$ Dent Res 2009;88:851-855.

This article is protected by copyright. All rights reserved. 
43. Yu Z, Zhai G, Singmann P, et al. Human serum metabolic profiles are age dependent. Aging Cell 2012;11:960-967.

44. Vignoli A, Tenori L, Luchinat C, Saccenti E. Age and sex effects on plasma metabolite association networks in healthy subjects. J Proteome Res 2018;17:97-107.

45. Hajishengallis G, Korostoff JM. Revisiting the Page \& Schroeder model: the good, the bad and the unknowns in the periodontal host response 40 years later. Periodontol 2000 2017;75:116-151.

46. Khoury MJ, Gwinn M, Ioannidis JP. The emergence of translational epidemiology: from scientific discovery to population health impact. Am J Epidemiol 2010;172:517-524.

\section{Figure legends}

Figure 1. PCA/CA score plot built on saliva spectra of healthy (HI) and periodontitis patients (GAgP and GCP). Healthy individuals are very well recognized, while the saliva samples of chronic and aggressive periodontitis are confounded within the same metabolic space as suggested by crossvalidation result.

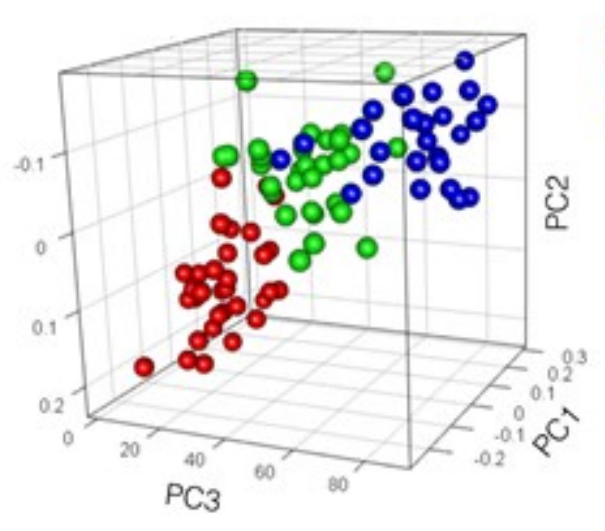

- HI (Healthy individuals)

- GAgP (Generalized Aggressive Periodontitis)

- GCP (Generalized Chronic Periodontitis)

Confusion matrix:

HI GCP GAgP

HI $\quad 71.3 \quad 24.8 \quad 3.9$

GCP 14.866 .419 .1

GAgP $10.7 \quad 61.2 \quad 27.8$

Prediction accuracy: $61 \%$

This article is protected by copyright. All rights reserved. 
Figure 2. PCA/CA score plots. Discrimination between saliva spectra of healthy individuals and chronic periodontitis patients (A) and between healthy and aggressive periodontitis patients (B). In both cases, the discrimination is effective as it arises from the related prediction accuracy.

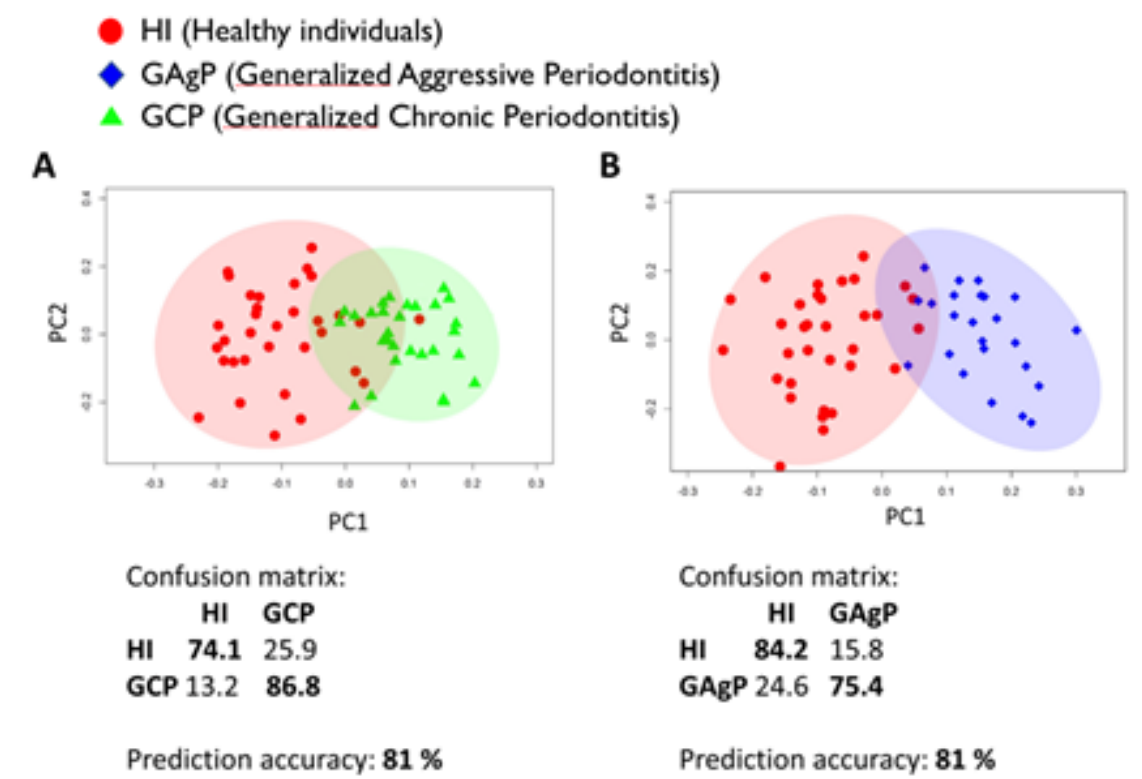

Figure 3. PCA/CA score plot. Discrimination between saliva spectra of chronic and aggressive periodontitis subjects. The model is not effective in discriminating the two groups $(60 \%$ predictive accuracy).

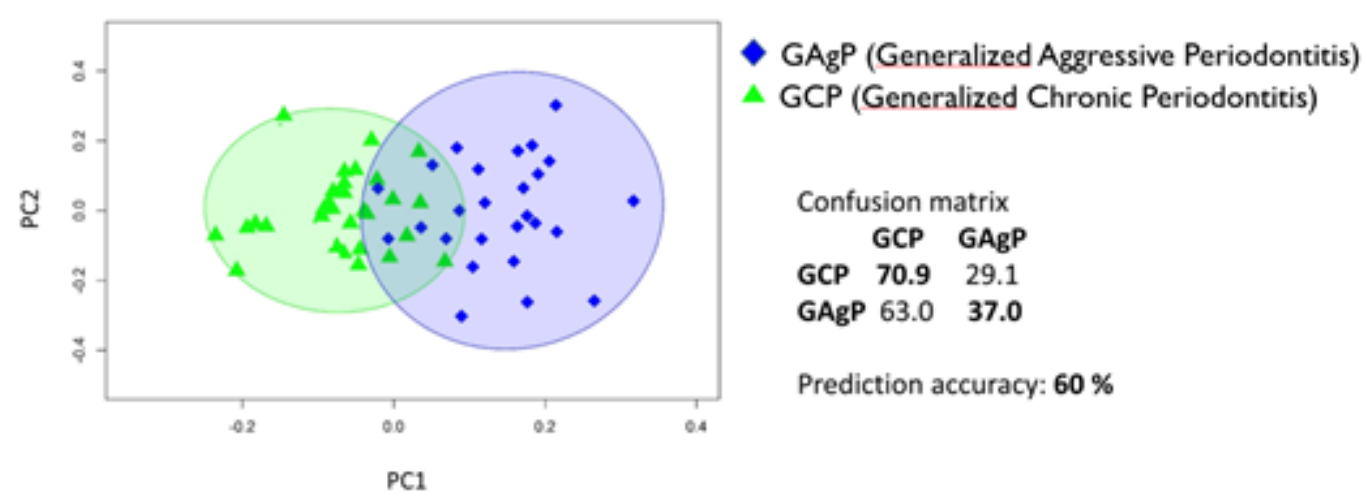

This article is protected by copyright. All rights reserved. 
Figure 4. Changes in metabolites levels between healthy individuals and chronic periodontitis (A) and between healthy and aggressive periodontitis (B) calculated as the $\log _{2}$ Fold Change (FC) ratio of the normalized median intensities of the corresponding signals in the spectra of the two groups. Green bars represent significantly altered metabolites $(P<0.05)$.

A

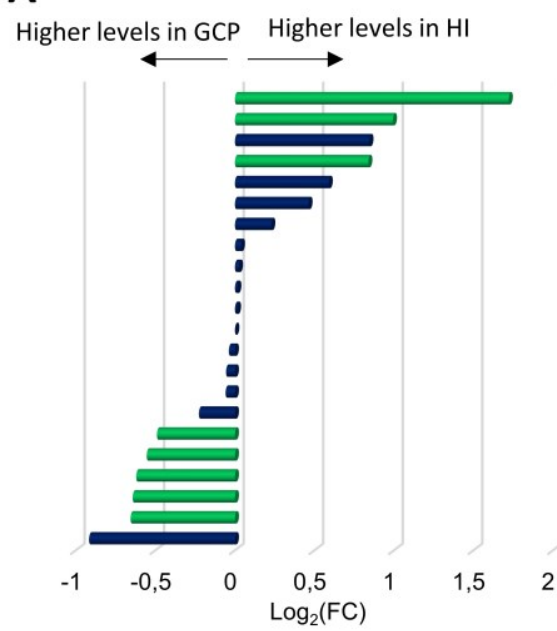

B

Pyruvate
N-acetyl groups
Isopropanol
Lactate
Ethanol
Sarcosine
Butyrate
GABA
Acetate
Methanol
Propionate
Methylamine
Succinate
Glycine
Choline
Alanine
Tyrosine
Valine
Isoleucine
Phenylalanine
Proline
Formate

Higher levels in GAgP Higher levels in $\mathrm{HI}$

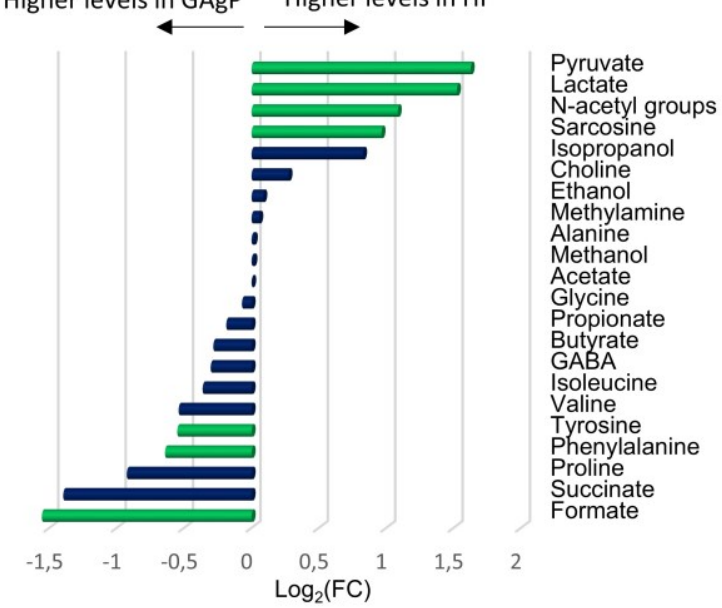


Table 1. Metabolites that result discriminant [median \pm mean absolute deviation (MAD), in arbitrary units] between healthy individuals (HI) and patients with GCP and GAgP.

\begin{tabular}{|c|c|c|c|c|c|}
\hline Variable & HI Group $(n=39)$ & GCP Group $(n=33)$ & FDR* HI vs. GCP & GAgP Group ( $n=28)$ & FDR* HI vs .GAgP \\
\hline Isoleucine & $0.131 \pm 0.064$ & $0.202 \pm 0.075$ & 0.0029 & $0.169 \pm 0.071$ & 0.4120 \\
\hline Valine & $0.079 \pm 0.04$ & $0.115 \pm 0.075$ & 0.0037 & $0.114 \pm 0.081$ & 0.166 \\
\hline Propionate & $0.598 \pm 0.207$ & $0.592 \pm 0.265$ & 0.9720 & $0.681 \pm 0.544$ & 0.3395 \\
\hline Isopropanol & $0.05 \pm 0.048$ & $0.028 \pm 0.03$ & 0.1308 & $0.028 \pm 0.041$ & 0.2916 \\
\hline Ethanol & $0.055 \pm 0.045$ & $0.037 \pm 0.032$ & 0.1999 & $0.052 \pm 0.053$ & 0.8935 \\
\hline Lactate & $0.249 \pm 0.62$ & $0.139 \pm 0.059$ & 0.0007 & $0.087 \pm 0.108$ & 0.0044 \\
\hline Alanine & $0.21 \pm 0.083$ & $0.245 \pm 0.107$ & 0.1920 & $0.207 \pm 0.152$ & 0.9922 \\
\hline Butyrate & $0.048 \pm 0.032$ & $0.041 \pm 0.031$ & 0.9031 & $0.058 \pm 0.043$ & 0.5957 \\
\hline Acetate & $13.503 \pm 3.046$ & $13.267 \pm 4.108$ & 0.9412 & $13.462 \pm 5.744$ & 0.8209 \\
\hline $\mathrm{N}$-acetyl-groups & $0.747 \pm 0.463$ & $0.375 \pm 0.229$ & 0.0086 & $0.352 \pm 0.451$ & 0.0481 \\
\hline Proline & $0.045 \pm 0.033$ & $0.071 \pm 0.046$ & 0.0221 & $0.085 \pm 0.059$ & 0.1171 \\
\hline Pyruvate & $0.272 \pm 0.343$ & $0.082 \pm 0.05$ & 0.0001 & $0.088 \pm 0.063$ & 0.0044 \\
\hline Succinate & $0.065 \pm 0.126$ & $0.067 \pm 0.082$ & 0.9720 & $0.171 \pm 0.124$ & 0.33956 \\
\hline Methylamine & $0.023 \pm 0.011$ & $0.023 \pm 0.011$ & 0.9031 & $0.022 \pm 0.011$ & 0.8714 \\
\hline Sarcosine & $0.026 \pm 0.02$ & $0.019 \pm 0.012$ & 0.2134 & $0.013 \pm 0.007$ & 0.0086 \\
\hline GABA & $0.128 \pm 0.066$ & $0.125 \pm 0.085$ & 0.9637 & $0.158 \pm 0.113$ & 0.6568 \\
\hline Choline & $0.197 \pm 0.103$ & $0.205 \pm 0.069$ & 0.9412 & $0.163 \pm 0.146$ & 0.8483 \\
\hline Methanol & $0.083 \pm 0.179$ & $0.082 \pm 0.07$ & 0.9720 & $0.083 \pm 0.177$ & 0.8209 \\
\hline Glycine & $0.626 \pm 0.205$ & $0.65 \pm 0.303$ & 0.1920 & $0.657 \pm 0.549$ & 0.6707 \\
\hline Tyrosine & $0.057 \pm 0.0210$ & $0.08 \pm 0.02$ & 0.0297 & $0.083 \pm 0.037$ & 0.0481 \\
\hline Phenylalanine & $0.092 \pm 0.0412$ & $0.143 \pm 0.044$ & 0.0007 & $0.1430 \pm 0.044$ & 0.0099 \\
\hline Formate & $0.0045 \pm 0.021$ & $0.009 \pm 0.0684$ & 0.1197 & $0.0134 \pm 0.073$ & 0.0086 \\
\hline
\end{tabular}

*False discovery rate correction. Bold face indicates statistically significant inter-group differences.

This article is protected by copyright. All rights reserved. 
Table 2. An integrated analysis based on MetaboAnalyst software: view of most contributing pathways.

\begin{tabular}{ccccc}
\hline Pathway Name & P-value & Holm P-value & FDR* & Impact \\
\hline Phenylalanine metabolism & $5.95 \times 10^{-5}$ & 0.005 & 0.005 & 0.12 \\
Pyruvate metabolism & 0.002 & 0.127 & 0.03 & 0.32 \\
\hline
\end{tabular}

*False discovery rate correction.

This article is protected by copyright. All rights reserved. 